\title{
Seleção de Plantas com Potencial para FitorRemediação de SOlOS CONTAMINADOS COM O HERBICIDA TRIFLOXYSUlFURON SODIUM ${ }^{1}$
}

\author{
Plant Selection with Potential for Phytoremediation of Soils Contaminated with \\ Trifloxysulfuron Sodium
}

\author{
PROCÓPIO, S.O. ${ }^{2}$, SANTOS, J.B. ${ }^{3}$, SILVA, A.A. ${ }^{4}$, PIRES, F.R. ${ }^{2}$, RIBEIRO JÚNIOR, J.I. ${ }^{5}$ \\ SANTOS, E.A. ${ }^{6}$ e FERREIRA, L.R. ${ }^{4}$
}

\begin{abstract}
RESUMO - Este trabalho teve como objetivo identificar plantas tolerantes ao trifloxysulfuron sodium, visando utilizá-las em programas de fitorremediação. Foram avaliadas dez espécies: Medicago sativa, Avena strigosa, Crotalaria juncea, Canavalia ensiformis, Helianthus annus, Dolichus lablab, Stylosantes guianensis, Mucuna deeringiana, Raphanus sativus e Eleusine indica. O delineamento experimental empregado foi o de blocos ao acaso, com parcelas subdivididas, sendo utilizadas três repetições. $\mathrm{O}$ fator aplicado na parcela principal foram as doses de trifloxysulfuron sodium $\left(0,00 ; 3,75 ; 7,50\right.$; e 15,00 $\left.\mathrm{g} \mathrm{ha}^{-1}\right)$ e, na subparcela, as épocas de avaliação [15, 30, 45 e 60 dias após a semeadura (DAS)]. Foram avaliados a altura de plantas, os sintomas de toxicidade e, aos 60 DAS, também a biomassa seca da parte aérea, das raízes e total das plantas. Verificou-se que todas as espécies sobreviveram à presença do trifloxysulfuron sodium no solo, mesmo quando aplicado em dose duas vezes maior que a recomendada $\left(15,00 \mathrm{~g} \mathrm{ha}^{-1}\right)$, porém $M$. deeringiana, $D$. lablab, $C$. juncea e S. guianenesis foram as espécies que apresentaram maior tolerância ao herbicida, indicando potencial para uso em programas de fitorremediação de trifloxysulfuron sodium em solos.
\end{abstract}

Palavras-chave: seletividade, fitotoxicidade, descontaminação, adubos verdes, plantas daninhas.

ABSTRACT - This study aimed to identify trifloxysulfuron sodium-selective plants to be used in phytoremediation programs for this herbicide in soil. Ten plants were evaluated: Medicago sativa, Avena strigosa, Crotalaria juncea, Canavalia ensiformis, Helianthus annus, Dolichus lablab, Stylosantes guianensis, Mucuna deeringiana, Raphanus sativus and Eleusine indica. A randomized block design with split-plots and three replications was used, with the plots being the doses of trifloxysulfuron sodium (0.00; 3.75; 7.50; and $15.00 \mathrm{~g} \mathrm{ha}^{-1}$ ) and the split-plots the evaluation times [15, 30, 45, and 60 days after sowing (DAS)]. Plant height and phytotoxicity visual symptoms were evaluated, and, at 60 DAS, dry biomass yield was recorded. It was verified that all species survived the presence of trifloxysulfuron sodium in soil, even when the herbicide was applied at $2 x$ the recommended dose $\left(15.00 \mathrm{~g} \mathrm{ha}^{-1}\right)$; however, the species M. deeringiana, D. lablab, C. juncea and S. guianenesis were the most tolerant to the herbicide, indicating potential for use in trifloxysulfuron sodium phytoremediation programs in soils.

Key words: selectivity, phytotoxicity, decontamination, green manure, weeds.

\section{INTRODUÇÃO}

O herbicida trifloxysulfuron sodium, recentemente registrado no País, vem sendo amplamente utilizado na cultura do algodão, sendo aplicado em pós-emergência inicial. Mesmo sendo um produto utilizado em baixas concentrações (em torno de 7,5 $\mathrm{g} \mathrm{ha}^{-1}$ ), o periodo de espera para o plantio de culturas sensiveis, recomendado pelo fabricante, é de

Recebido para publicação em 6.6.2003 e na forma revisada em 5.3.2004.

2 Prof. do Dep. de Fitossanidade da Universidade Federal de Pelotas - UFPEL. ${ }^{3}$ Doutorando, Dep. de Fitotecnia da Universidade Federal de Viçosa - UFV, 36570-000 Viçosa-MG; ${ }^{4}$ Prof. do Dep. de Fitotecnia da UFV; ${ }^{5}$ Prof. do Dep. de Informática da UFV; ${ }^{6}$ Acadêmico de Agronomia - UFV. 
nove meses a contar de sua aplicação. Esse fato pode limitar sua utilização em áreas onde o agricultor cultiva, por exemplo, feijão no inverno ou semeia milho na primavera.

Outro fator negativo relacionado à utilização de herbicidas de longo efeito residual no solo é a maior probabilidade de ocorrência de lixiviação de suas moléculas originais ou de seus metabólitos para camadas mais profundas no perfil do solo, podendo atingir o lençol freático.

A fitorremediação é uma técnica que pode acelerar a retirada de compostos tóxicos, como herbicidas, do solo, promovendo sua descontaminação (Cunningham et al., 1996). Baseia-se na seletividade que algumas espécies vegetais exibem a determinado composto específico e/ ou a um mecanismo de ação. A tolerância pode ser resultante de processos como a translocação diferencial de compostos orgânicos para outros tecidos da planta, com subseqüente volatilização, ou da degradação parcial ou completa, com transformação em compostos menos tóxicos, combinados e/ou ligados nos tecidos das plantas (Accioly \& Siqueira, 2000). Em geral, a maioria dos compostos orgânicos passa por alguma transformação nas células das plantas antes de ser isolada em vacúolos ou ligar-se a estruturas celulares insolúveis, como a lignina (Macek et al., 2000). Outra possibilidade é a fitoestimulação, na qual, em razão da liberação de exsudatos radiculares, há o estímulo à atividade microbiana, que atua degradando o composto no solo, o que caracteriza, em algumas plantas, a aptidão rizosférica para a biorremediação de compostos tóxicos (Cunningham et al., 1996). Diversos trabalhos descrevem a possibilidade da utilização de plantas na descontaminação de solos (Anderson \& Coats, 1995; Wagner \& Zablotowicz, 1997; Kruger et al., 1997; Hoagland et al., 1997; Boyle \& Shann, 1998).

A seleção de plantas que apresentem tolerância ao pesticida é o primeiro passo na seleção de espécies potencialmente fitorremediadoras. Contudo, deve-se evitar a utilização de espécies de dificil controle posterior e, se possivel, selecionar espécies que promovam outros beneficios ao solo, como é o caso dos adubos verdes. No entanto, não existem até o momento relatos de pesquisas sobre a tolerância de leguminosas de cobertura do solo ao herbicida trifloxysulfuron sodium.

Este trabalho teve como objetivo selecionar espécies vegetais tolerantes ao trifloxysulfuron sodium, visando utilizá-las em programas de fitorremediação.

\section{MATERIAL E MÉTODOS}

O experimento foi realizado em vasos e conduzido em casa de vegetação. O substrato utilizado foi um solo classificado como Argissolo Vermelho-Amarelo, de textura argilo-arenosa, cuja caracterização físico-química encontra-se na Tabela 1.

Por se tratar de um herbicida relativamente novo no mercado brasileiro, poucas informações se encontram disponiveis sobre a tolerância de espécies vegetais a esse produto. Devido a esse fato, o critério de seleção inicial baseou-se em três itens: facilidade na aquisição de sementes; beneficio adicional ao solo; e fácil controle posterior. As espécies testadas foram:

Tabela 1 - Composição físico-química da camada arável $(0-20 \mathrm{~cm})$ do solo Argissolo Vermelho-Amarelo utilizado no experimento

\begin{tabular}{|c|c|c|c|c|c|c|c|c|c|c|}
\hline \multicolumn{11}{|c|}{ Análise Granulométrica ( dag kg $\left.^{-1}\right)$} \\
\hline & Argila & \multicolumn{2}{|c|}{ Silte } & \multicolumn{2}{|c|}{ Areia Fina } & \multicolumn{2}{|c|}{ Areia Grossa } & \multicolumn{3}{|c|}{ Classificação Textural } \\
\hline & 39 & \multicolumn{2}{|c|}{11} & \multicolumn{2}{|c|}{17} & \multicolumn{2}{|r|}{33} & \multicolumn{3}{|c|}{ Argilo-arenosa } \\
\hline \multicolumn{11}{|c|}{ Análise Química } \\
\hline $\mathrm{pH}$ & $\mathrm{P}$ & $\mathrm{H}+\mathrm{Al}$ & $\mathrm{Al}^{3+}$ & $\mathrm{Ca}^{2+}$ & $\mathrm{Mg}^{2+}$ & $\mathrm{K}^{+}$ & $\mathrm{CTC}_{\text {total }}$ & $\mathrm{V}$ & $\mathrm{m}$ & MO \\
\hline $\mathrm{H}_{2} \mathrm{O}$ & $\left(\mathrm{mg} \mathrm{dm}^{-3}\right)$ & \multicolumn{4}{|c|}{$\left(\mathrm{cmol}_{\mathrm{c}} \mathrm{dm}^{-3}\right)$} & $\left(\mathrm{mg} \mathrm{dm}^{-3}\right)$ & $\left(\mathrm{cmol}_{\mathrm{c}} \mathrm{dm}^{-3}\right)$ & \multicolumn{2}{|c|}{$(\%)$} & $\left(\right.$ dag $\left.\mathrm{kg}^{-1}\right)$ \\
\hline 5,8 & 1,4 & 4,3 & 0,0 & 3,8 & 1,4 & 123 & 9,81 & 56 & 0 & 2,18 \\
\hline
\end{tabular}

* Análises realizadas nos Laboratórios de Análises Físicas e Químicas de Solo do Departamento de Solos da UFV, segundo a metodologia da Empresa Brasileira de Pesquisa Agropecuária-EMBRAPA (1997). 
Medicago sativa (alfafa), Avena strigosa (aveiapreta), Crotalaria juncea (crotalária), Canavalia ensiformis (feijão-de-porco), Helianthus annus (girassol), Dolichus lablab (lablabe), Stylosantes guianensis (mineirão), Mucuna deeringiana (mucuna-anã), Raphanus sativus (nabo-forrageiro) e Eleusine indica (capim-pé-de-galinha).

A unidade experimental foi constituída de um vaso de polietileno, contendo $3 \mathrm{dm}^{3}$ de solo. Após o enchimento e umedecimento dos vasos, procedeu-se à aplicação em pré-emergência do herbicida empregando pulverizador costal pressurizado com gás carbônico $\left(\mathrm{CO}_{2}\right)$, composto de barra com duas pontas de pulverização em leque 110.03 , espaçadas de $0,50 \mathrm{~m}$, com volume de aplicação de $260 \mathrm{~L} \mathrm{ha}^{-1}$.

A semeadura das espécies selecionadas nos vasos foi realizada no dia seguinte à aplicação do trifloxysulfuron sodium. Após a aplicação dos tratamentos, os vasos foram mantidos sob irrigação, a fim de se conservar a umidade do solo em torno de $80 \%$ da capacidade de campo.

As características avaliadas para determinação da seletividade do herbicida às diferentes espécies foram os sintomas de toxicidade, avaliada visualmente, atribuindo-se notas de acordo com os sintomas de intoxicação na parte aérea das plantas, utilizando escala variando de 0 a $100 \%$, para ausência de sintomas até a morte da planta, respectivamente; e a altura de plantas, adotando-se como base para medição o meristema apical nas dicotiledôneas e a extremidade da folha mais alta nas demais espécies. O delineamento experimental utilizado foi o de blocos casualizados em parcelas subdivididas, com três repetições para cada espécie vegetal. O fator da parcela principal foi a dose do trifloxysulfuron sodium $(0,00$; 3,$75 ; 7,50$; e $15,00 \mathrm{~g} \mathrm{ha}^{-1}$ ) e o fator da subparcela a época de avaliação [15, 30, 45 e 60 dias após a semeadura (DAS)]. Na última avaliação, realizada aos 60 DAS, avaliaram-se, além dos sintomas de toxicidade e da altura das plantas, a biomassa seca da parte aérea, a biomassa seca de raizes e a biomassa seca total, obtidas por meio de pesagem do material colhido, secado em estufa de circulação forçada $\left(70 \pm 2{ }^{\circ} \mathrm{C}\right)$ por 72 horas. Nesta avaliação adotou-se o delineamento em blocos casualizados com três repetições, em que foram estudadas as quatro doses do trifloxysulfuron sodium.
Todas as variáveis que atenderam às pressuposições de normalidade e homogeneidade das variâncias, por meio dos testes de Lilliefors e de Cochran, respectivamente, foram submetidas à análise de variância para a verificação de significância dos dois fatores estudados e da interação entre eles pelo teste F, a 5\% de significância. Os efeitos das doses do trifloxysulfuron sodium e das épocas de avaliações foram estudados por meio de análise de regressão, a 1 ou $5 \%$ de significância.

\section{RESULTADOS E DISCUSSÃO}

\section{Altura de plantas}

Para as espécies vegetais $M$. sativa, $A$. strigosa, C. ensiformis, $H$. annus, $R$. sativus e E. indica a altura das plantas aumentou $(\mathrm{P}<0,01)$ ao longo das épocas de avaliação (Figura 1), sendo suas equações de regressão apresentadas na Tabela 2. Por outro lado, ela diminuiu $(\mathrm{P}<0,01$ ou $\mathrm{P}<0,05)$ com o aumento das doses do trifloxysulfuron sodium. No entanto, A. strigosa e C. ensiformis apresentaram maior recuperação da ação fitotóxica dessa sulfoniluréia, em relação a esse parâmetro avaliado ao longo do tempo (Figura 1).

As espécies C. juncea, D. lablab, $M$. deeringiana e $S$. guianensis apresentaram aumento da altura com o decorrer do tempo (Figura 2), porém não sofreram efeito das doses do trifloxysulfuron sodium, como evidenciado nas suas equações (Tabela 2). Dal Piccolo \& Christoffoleti (1985) constataram inibição no desenvolvimento de C. juncea até 13 meses após a aplicação do herbicida tebuthiuron.

\section{Sintomas de toxicidade}

Constatou-se (Figuras 3 e 4 e Tabela 2) que os sintomas de toxicidade observados nas espécies A. strigosa, C. ensiformis, $S$. guianensis, $R$. sativus, $E$. indica, $M$. sativa e H. annus diminuíram $(\mathrm{P}<0,01)$ ao longo do tempo (aumento das épocas de avaliações). Para as demais espécies não foi detectado efeito das épocas de avaliações sobre essa característica. Também, percebe-se (Figura 3) que o aumento da dose do herbicida ocasionou incremento linear na intensidade dos sintomas de toxicidade para as espécies A. strigosa, C. ensiformis, S. guianensis, $R$. sativus e 

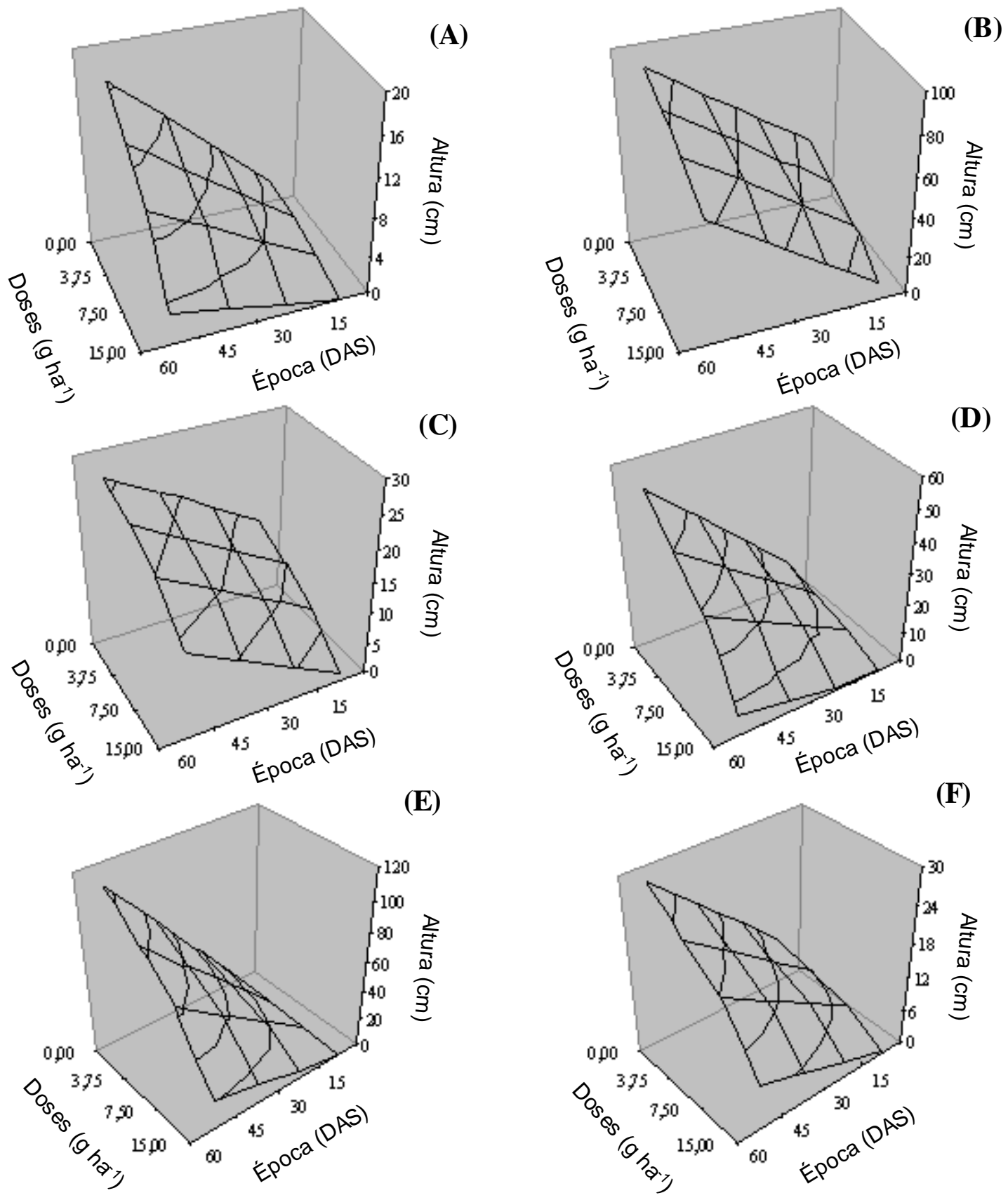

Figura 1 - Estimativas da altura de plantas de Medicago sativa (A), Avena strigosa (B), Canavalia ensiformis (C), Helianthus annus (D), Raphanus sativus (E) e Eleusine indica (F) em função da época de avaliação [dias após a semeadura (DAS)] e de doses de trifloxysulfuron sodium $\left(\mathrm{g} \mathrm{ha}^{-1}\right)$.

E. indica, sendo a intensidade do incremento variável conforme a época e a planta. De maneira geral, observa-se que, com a aplicação da dose comercial do trifloxysulfuron sodium $\left(7,5 \mathrm{~g} \mathrm{ha}^{-1}\right)$ aos $15 \mathrm{DAS}$, os valores dos sintomas de toxicidade variaram de $40 \%$ para $C$. ensiformis a pouco mais de $60 \%$ para $E$. indica, sendo tais valores considerados elevados e, conseqüentemente, as plantas pouco tolerantes ao herbicida. Entretanto, aos 60 DAS a espécie S. guianensis apresentou boa recuperação dos sintomas (Figura 3). 
Seleção de plantas com potencial para fitorremediação ...

Tabela 2 - Estimativas dos efeitos de doses de trifloxysulfuron sodium (D) e das épocas de avaliação (E) sobre a altura de plantas e os sintomas de toxicidade em dez espécies vegetais avaliadas para fins de fitorremediação. Viçosa-MG, 2002

\begin{tabular}{|c|c|c|c|c|}
\hline \multirow[b]{2}{*}{ Espécie vegetal } & \multicolumn{2}{|l|}{ Altura de plantas } & \multicolumn{2}{|l|}{ Sintomas de toxicidade } \\
\hline & Equação de regressão & $\begin{array}{c}\mathrm{R}^{2} \\
(\%)\end{array}$ & Equação de regressão & $\begin{array}{l}\mathrm{R}^{2} \\
(\%)\end{array}$ \\
\hline M. sativa & $\hat{\mathrm{Y}}=-2,250+0,044 * \mathrm{D}+0,313 * * \mathrm{E}-0,015 * \mathrm{DE}$ & 61,56 & $\hat{\mathrm{Y}}=109,000-0,614^{* *} \mathrm{E}$ & 93,56 \\
\hline A. strigosa & $\hat{\mathrm{Y}}=16,612-1,699^{*} \mathrm{D}+1,201 * * \mathrm{E}$ & 95,09 & $\hat{\mathrm{Y}}=23,611+3,175 * * \mathrm{D}-0,472 * * \mathrm{E}$ & 84,54 \\
\hline C. juncea & $\hat{\mathrm{Y}}=-3,083+0,773^{* *} \mathrm{E}$ & 99,75 & $\bar{Y}=43,139$ & - \\
\hline C. ensiformis & $\hat{\mathrm{Y}}=8,827-0,805^{* *} \mathrm{D}+0,285^{* *} \mathrm{E}$ & 82,46 & $\hat{\mathrm{Y}}=-17,431+7,325 * * \mathrm{D}-0,174 * * \mathrm{E}$ & 98,93 \\
\hline H. annus & $\hat{\mathrm{Y}}=-3,283-0,081 * * \mathrm{D}+0,903 * * \mathrm{E}-0,047 * \mathrm{DE}$ & 82,81 & $\hat{\mathrm{Y}}=87,167-0,346^{* *} \mathrm{E}$ & 99,98 \\
\hline D. lablab & $\hat{\mathrm{Y}}=1,750+0,298 * * \mathrm{E}$ & 94,65 & $\overline{\mathrm{Y}}=24,722$ & - \\
\hline S. guianensis & $\hat{\mathrm{Y}}=-1,396+0,204 * * \mathrm{E}$ & 96,58 & $\hat{\mathrm{Y}}=8,333+6,444 * \mathrm{D}-0,294 * * \mathrm{E}-0,062 * \mathrm{DE}$ & 90,38 \\
\hline M. deeringiana & $\hat{\mathrm{Y}}=7,000+0,134 * * \mathrm{E}$ & 95,38 & $\bar{Y}=7,639$ & - \\
\hline R. sativus & $\hat{\mathrm{Y}}=-30,267+1,336 * * \mathrm{D}+2,282 * * \mathrm{E}-0,118 * \mathrm{DE}$ & 76,21 & $\hat{\mathrm{Y}}=7,375+7,151 * * \mathrm{D}-0,549 * * \mathrm{E}$ & 97,02 \\
\hline E. indica & $\hat{\mathrm{Y}}=1,083-0,219^{* *} \mathrm{D}+0,442 * * \mathrm{E}-0,018 * \mathrm{DE}$ & 89,07 & $\hat{\mathrm{Y}}=44,514+3,389 * * \mathrm{D}-0,537 * * \mathrm{E}$ & 72,19 \\
\hline
\end{tabular}

** Significativo pelo teste $\mathrm{t}$ a $1 \%$ de probabilidade. $*$ Significativo pelo teste $\mathrm{t}$ a $5 \%$ de probabilidade.

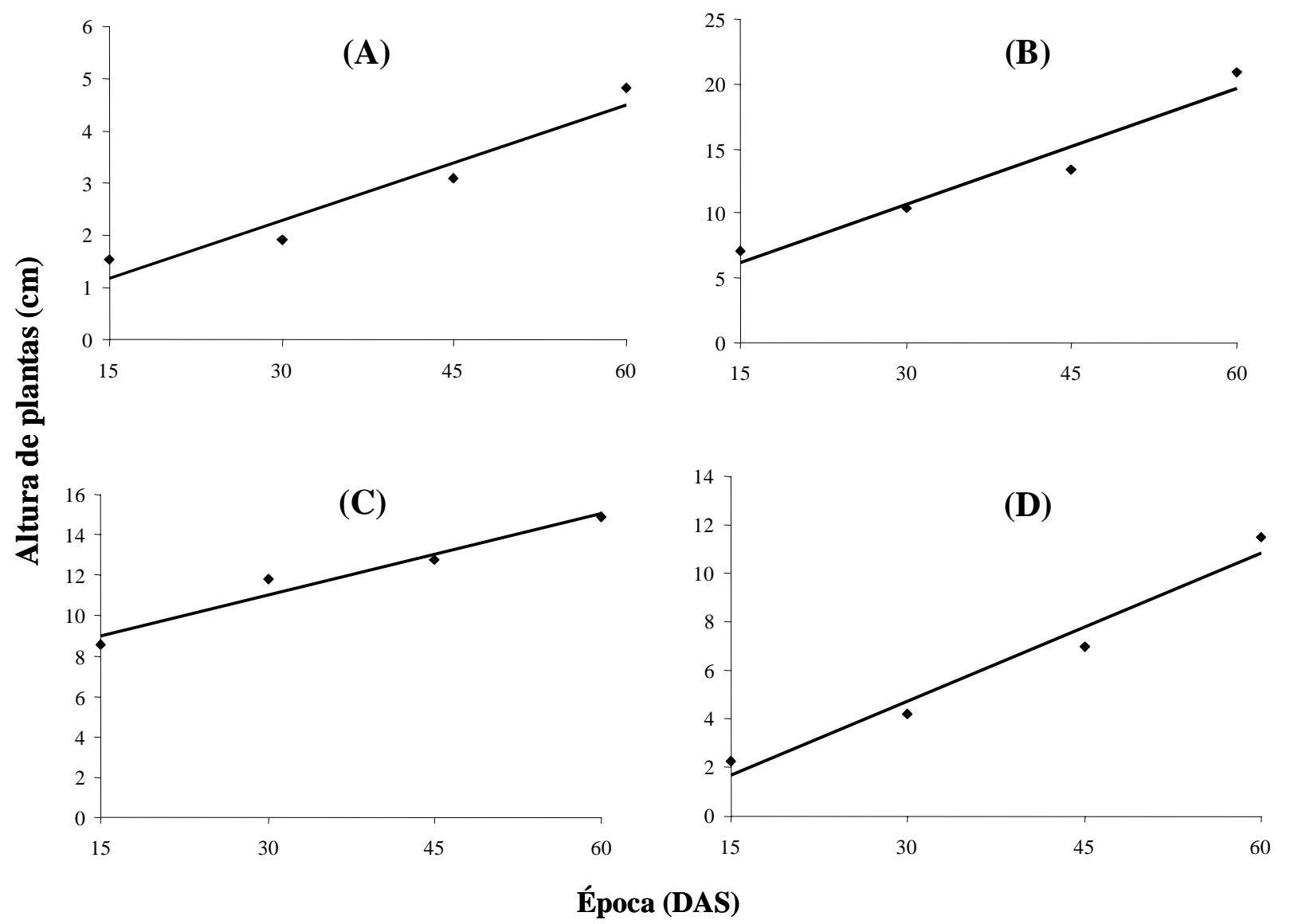

Figura 2 - Estimativas da altura de plantas de Crotalaria juncea (A), Dolichus lablab (B), Mucuna deeringiana (C) e Stylosantes guianensis (D) em função da época de avaliação [dias após a semeadura (DAS)]. 

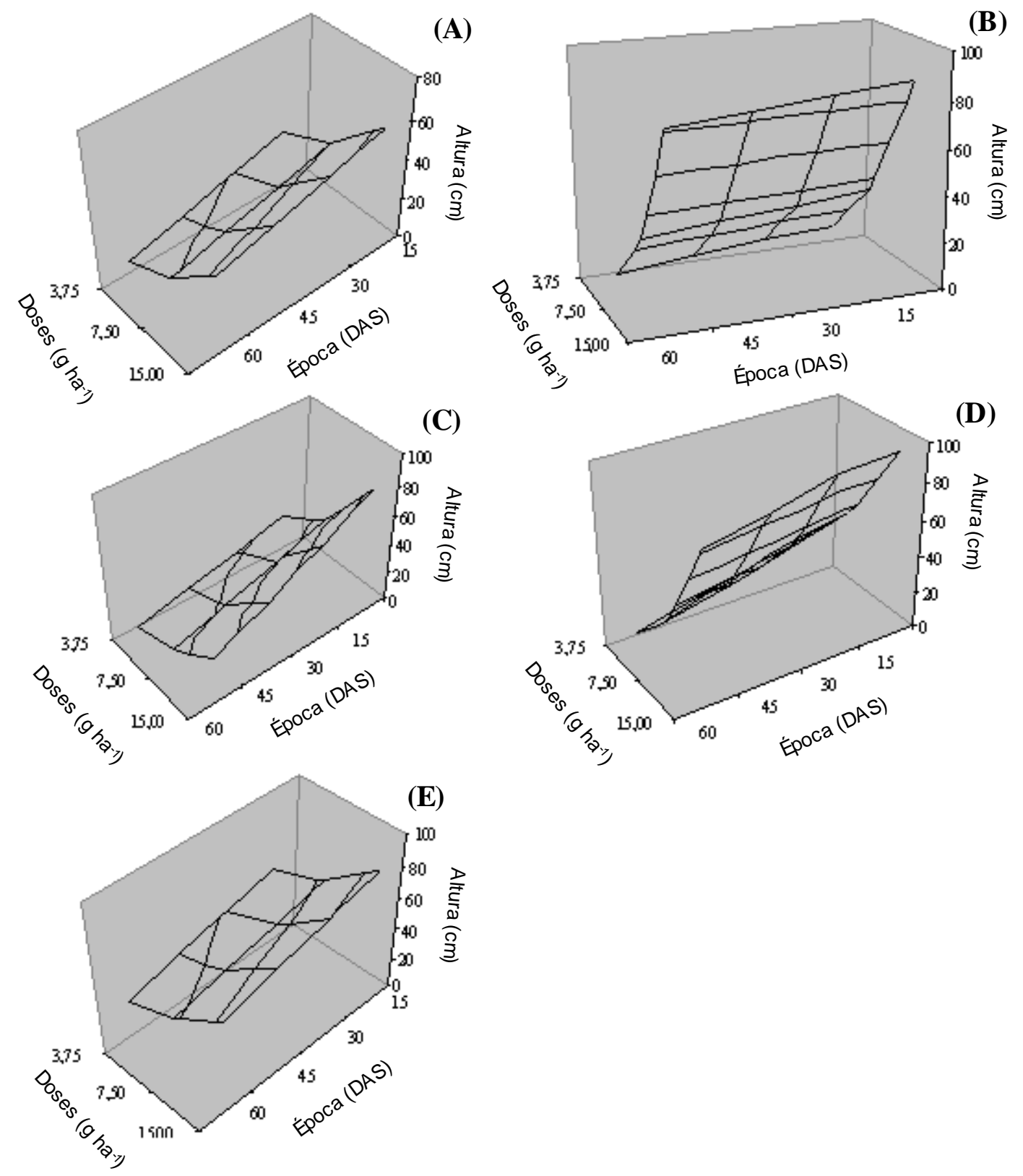

(D)

Figura 3 - Estimativas dos sintomas de toxicidade apresentados por Avena strigosa (A), Canavalia ensiformis (B), Stylosantes guianensis (C), Raphanus sativus (D) e Eleusine indica (E) em função da época de avaliação [dias após a semeadura (DAS)] e de doses de trifloxysulfuron sodium $\left(\mathrm{g} \mathrm{ha}^{-1}\right)$.

Apesar de não ter sido observado efeito $(\mathrm{P}<0,05)$ das doses do trifloxysulfuron sodium sobre as espécies $M$. sativa e $H$. annus, estas plantas se mostraram altamente sensiveis ao herbicida (Figura 4), apresentando sintomas de toxicidade variando da ordem de 80 a quase $100 \%$ aos 15 DAS, com pequena diminuição dos sintomas tóxicos ao longo do tempo.
Não foi detectado efeito tanto das doses de trifloxysulfuron sodium como das épocas de avaliação em relação a $C$. juncea, D. lablab e $M$. deeringiana. No entanto, a menor média dos sintomas de toxicidade foi constatada em $M$. deeringiana $(7,6 \%)$, a qual, conseqüentemente, se mostrou a mais tolerante ao herbicida quanto a essa característica. Silva \& Bueno (2000) 
verificaram que Mucuna aterrima foi tolerante aos herbicidas alachlor, imazaquin e pendimenthalin, sendo suscetivel apenas ao 2,4-DB.

\section{Biomassa seca das plantas}

Observa-se, pela análise da Tabela 3, que apenas houve efeito significativo das doses do trifloxysulfuron sodium sobre a biomassa seca da parte aérea (BSPA) e a biomassa seca das raízes (BSR) nas espécies A. strigosa, C. ensiformis e E. indica. Em relação à biomassa seca total (BST), além dessas espécies citadas, inclui-se $M$. sativa no grupo de plantas que apresentaram diminuição da biomassa com o aumento das doses do herbicida (Figura 5).

Verificou-se, para as condições do experimento, que todas as espécies sobreviveram à presença do trifloxysulfuron sodium no solo, mesmo quando aplicado em dose duas vezes maior que a recomendada pelo fabricante $\left(15,00 \mathrm{~g} \mathrm{ha}^{-1}\right)$. Todavia, realizando análise integrada dos resultados, as espécies $M$. deeringiana, D. lablab, C. juncea e $S$. guianenesis apresentaram-se mais tolerantes ao herbicida, indicando maior potencial para a continuação dos estudos de fitorremediação de trifloxysulfuron sodium em solos.

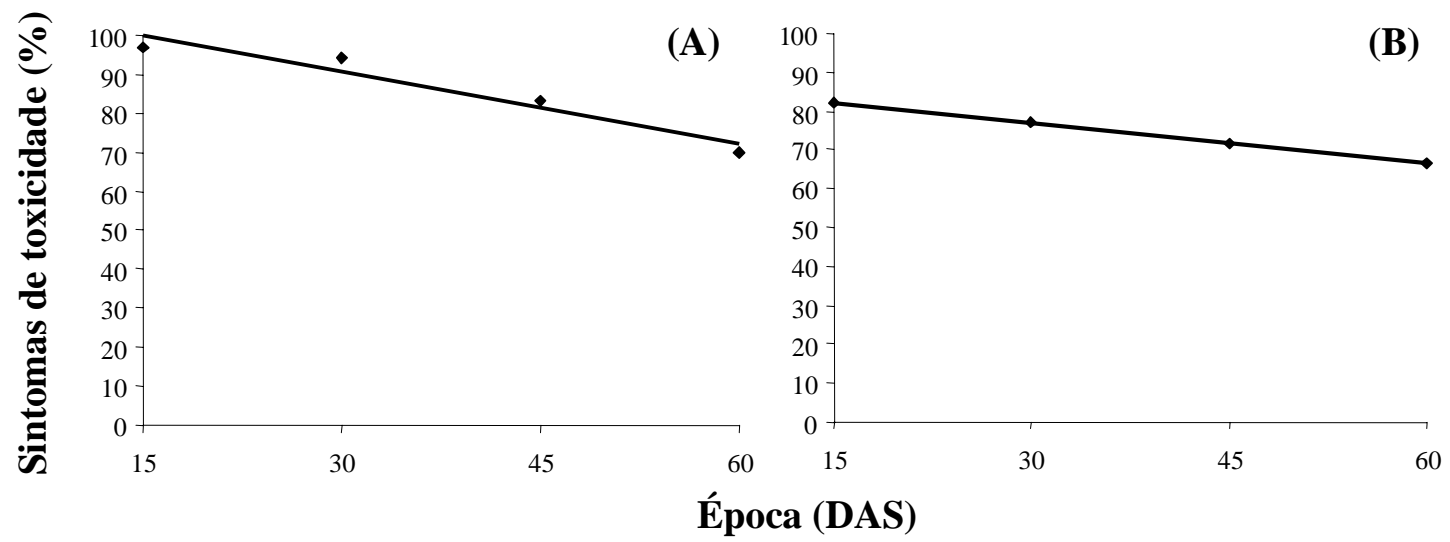

Figura 4 - Estimativas dos sintomas de toxicidade apresentados por Medicago sativa (A) e Helianthus annus (B) em função da época de avaliação [dias após a semeadura (DAS)].

Tabela 3 - Estimativas dos efeitos de doses de trifloxysulfuron sodium (D) sobre a biomassa seca de parte aérea (BSPA), das raízes (BSR) e total (BST) em dez espécies vegetais avaliadas para fins de fitorremediação. Viçosa-MG, 2002

\begin{tabular}{|c|c|c|c|c|c|c|}
\hline \multirow[b]{2}{*}{ Espécie vegetal } & \multicolumn{2}{|l|}{ BSPA } & \multicolumn{2}{|l|}{ BSR } & \multicolumn{2}{|l|}{ BST } \\
\hline & Equação de regressão & $\begin{array}{c}\mathrm{R}^{2} \\
(\%)\end{array}$ & Equação de regressão & $\begin{array}{l}\mathrm{R}^{2} \\
(\%)\end{array}$ & Equação de regressão & $\begin{array}{c}\mathrm{R}^{2} \\
(\%)\end{array}$ \\
\hline M. sativa & $\bar{Y}=0,207$ & - & $\bar{Y}=0,091$ & - & $\hat{\mathrm{Y}}=0,476-0,0270 * \mathrm{D}$ & 54,8 \\
\hline A. strigosa & $\hat{\mathrm{Y}}=2,669-0,1049^{*} \mathrm{D}$ & 69,02 & $\hat{\mathrm{Y}}=2,240-0,1375^{* *} \mathrm{D}$ & 79,60 & $\hat{\mathrm{Y}}=4,910-0,242 * * \mathrm{D}$ & 75,8 \\
\hline C. juncea & $\bar{Y}=0,973$ & - & $\bar{Y}=0,502$ & - & $\overline{\mathrm{Y}}=1,474$ & - \\
\hline C. ensiformis & $\hat{\mathrm{Y}}=3,995-0,1830^{* *} \mathrm{D}$ & 67,76 & $\hat{\mathrm{Y}}=3,093-0,1364 * * \mathrm{D}$ & 83,00 & $\hat{\mathrm{Y}}=7,088-0,319 * * \mathrm{D}$ & 75,3 \\
\hline H. annus & $\bar{Y}=1,459$ & - & $\bar{Y}=1,034$ & - & $\overline{\mathrm{Y}}=2,494$ & - \\
\hline D. lablab & $\bar{Y}=1,643$ & - & $\overline{\mathrm{Y}}=1,025$ & - & $\bar{Y}=2,668$ & - \\
\hline S. guianensis & $\bar{Y}=0,233$ & - & $\bar{Y}=0,097$ & - & $\bar{Y}=0,330$ & - \\
\hline M. deeringiana & $\overline{\mathrm{Y}}=2,063$ & - & $\overline{\mathrm{Y}}=1,076$ & - & $\overline{\mathrm{Y}}=3,139$ & - \\
\hline R. sativus & $\bar{Y}=2,183$ & - & $\bar{Y}=1,419$ & - & $\bar{Y}=3,602$ & - \\
\hline E. indica & $\hat{\mathrm{Y}}=1,139-0,0526^{* *} \mathrm{D}$ & 56,34 & $\hat{\mathrm{Y}}=0,584-0,0249^{* *} \mathrm{D}$ & 43,21 & $\hat{\mathrm{Y}}=1,724-0,07751 * * \mathrm{D}$ & 54,2 \\
\hline
\end{tabular}

** Significativo pelo teste $\mathrm{t}$ a $1 \%$ de probabilidade. * Significativo pelo teste $\mathrm{t}$ a $5 \%$ de probabilidade. 


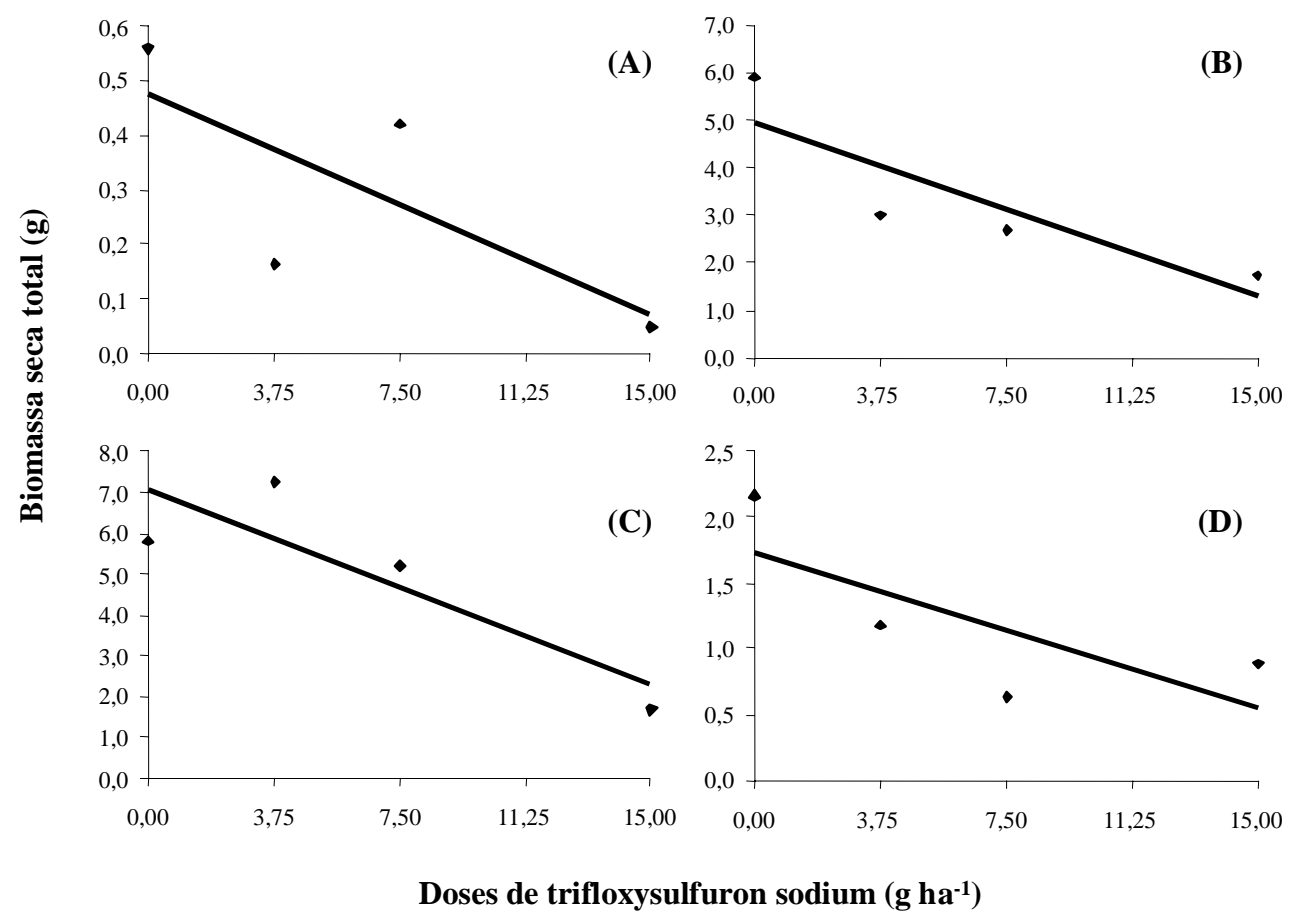

Figura 5 - Estimativas da biomassa seca total de plantas de Medicago sativa (A), Avena strigosa (B), Canavalia ensiformis (C) e Eleusine indica (D) em função de doses de trifloxysulfuron sodium $\left(\mathrm{g} \mathrm{ha}^{-1}\right)$.

\section{AGRADECIMENTOS}

À empresa Syngenta Proteção de Cultivos Ltda., pelo apoio financeiro.

\section{LITERATURA CITADA}

ACCIOLY, A. M. A.; SIQUEIRA, J. O. Contaminação química e biorremediação do solo. In: NOVAIS, R. F.; ALVAREZ V., V. H.; SCHAEFER, C. E. G. R. Tópicos em ciência do solo.' Viçosa, Sociedade Brasileira de Ciência do Solo, 2000. v. 1. p. 299-352.

ANDERSON, T. A.; COATS, J. R. Screening rhizosphere soil samples for the ability to mineralize elevated concentrations of atrazine and metolachlor. J. Environ. Sci. Health, B30: p. 473-484, 1995.

BOYLE, J. J.; SHANN, J. R. The influence of planting and soil characteristics on mineralization of 2,4,5-T in rhizosphere soil. J. Environ. Qual., v. 27, p. 704-709, 1998.

CUNNINGHAM, S. D.; ANDERSON, T. A.; SCHWAB, A. P. Phytoremediation of soils contaminated with organic pollutants. Adv. Agron., v. 56, p. 55-114, 1996.

DAL PICCOLO, C. R.; CHRISTOFFOLETI, P. J. Efeito residual de herbicidas utilizados na cultura da cana-deaçúcar sobre a Crotalaria juncea L. em rotação. Saccharum, v. 8, p. 34-38, 1985.
HOAGLAND, R. E.; ZABLOTOWICZ, R. M.; LOCKE, M. A. An integrated phytoremediaton strategy for chloracetamide herbicides in soil. In: Phytoremediation of soil and water contaminants, 1997, Washington, DC: Symposium Series... Washington, DC: American Chemical Society, 1997. p. 92-105.

KRUGER, E. L. et al. Atrazine degradation in pesticidecontaminated soils: phytoremediation potential. In: PHYTOREMEDIATION OF SOIL AND WATER CONTAMINANTS, 1997, Washington, DC. Symposium Series... Washington, DC: American Chemical Society, 1997. p. 54-64.

MACEK, T.; MACKOVÁ, M.; KÁŠ, J. Exploitation of plants for the removal of organics in environmental remediation. Biotechnol. Adv., v. 18, p. 23-34, 2000.

SILVA, J. F.; BUENO, C. R. Tolerância de leguminosas de cobertura do solo a herbicidas. I. In: CONGRESSO BRASILEIRO DA CIÊNCIA DAS PLANTAS DANINHAS, 22., 2000, Foz do Iguaçu. Resumos... Foz do Iguaçu: Sociedade Brasileira da Ciência das Plantas Daninhas, 2000. p. 375.

WAGNER, S. C.; ZABLOTOWICZ, R. M. Utilization of plant material for remediation of herbicide-contaminated soils. In: Phytoremediation of soil and water contaminants, 1997, Washington, DC. Symposium Series... Washington, DC: American Chemical Society, 1997. p. 65-76. 